\title{
Katugampola-type fractional differential equations with delay and impulses
}

\begin{abstract}
Our aim in this note is to study the existence of solutions of a Katugampola-type fractional impulsive differential equation with delay. We use successive approximation method to show the existence of solutions. In the end, an example is given to verify the hypothetical results.
\end{abstract}

Keywords: katugampola fractional derivative, impulsive equations, time delay
Volume I Issue 3 - 2018

\author{
M Janaki,' EM Elsayed, ${ }^{2,3}$ K Kanagarajan' \\ 'Department of Mathematics, Sri Ramakrishna Mission Vidyalaya \\ College of Arts and Science, India \\ ${ }^{2}$ Department of Mathematics, King Abdulaziz University, Saudi \\ Arabia \\ ${ }^{3}$ Department of Mathematics, Mansoura University, Egypt
}

Correspondence: Elsayed EM, Department of Mathematics, Faculty of Science, Mansoura University, Mansoura 355I6, Egypt, Email emelsayed@mans.edu.eg

Received: February 03, 2018| Published: May 01, 2018

\section{Introduction}

Because of its wide applicability in biology, medicine and in more and more fields, the theory of fractional differential equations(FDEs) has recently been attracting increasing interest, see for instance ${ }^{1-8}$ and references therein. Impulsive differential equations have played an important role in modelling phenomena, especially in describing dynamics of populations subject to abrupt changes as well as other phenomena such as harvesting, diseases, and so forth, some authors have used impulsive differential systems to describe the model since the last century. For the basic theory on impulsive differential equations, the reader can refer to the books ${ }^{9-12}$ and the papers. ${ }^{1,13-16}$ In addition, some modelling is done via impulsive functional differential equations when these processes involve hereditary phenomena such as biological and social macrosystems. For fractional functional differential equations, the initial value problem, for a class of nonlinear fractional functional differential equations is discussed. For more details, see. ${ }^{17-24}$ Motivated by the papers, ${ }^{25,26}$ the aim of this note is to discuss the existence and uniqueness of solutions of Katugampolatype FDEs with delay and impulses.

Consider the Katugampola-type FDEs with delay and impulse of the form,

$$
\left\{\begin{array}{c}
{ }_{c}^{\rho} D^{\omega} \mathfrak{Z}(t)=\mathfrak{H}\left(t, \mathfrak{Z}_{t}\right), t \neq t_{k} ; t \in \mathfrak{I}:=[0, T] ; \\
0^{+} \\
\Delta \mathfrak{Z}\left(t_{k}\right)=I_{k}\left(\mathfrak{Z}\left(t_{k}\right)\right), k=1,2, \ldots, m ; \\
\mathfrak{Z}(t)=\psi(t), t \in[-\mu, 0],
\end{array}\right.
$$

where ${ }_{c}^{\rho} D^{\omega}$ is the generalized fractional derivative in Caputo sense, $\omega \in \mathbb{R}^{+}, 0^{+}>0,0=t_{0}<t_{1}<t_{2}<\ldots<t_{m}<t_{m+1}=T, \wp \in \mathfrak{C}(\mathfrak{J} \times \mathbb{R}, \mathbb{R})$ and $I_{k} \in \mathfrak{C}(\mathbb{R}, \mathbb{R})$ are given functions satisfying some assumptions that will be specified later. $\Delta\left(\mathfrak{Z}\left(t_{k}\right)\right)=\mathfrak{Z}\left(t_{k}^{+}\right)-\mathfrak{Z}\left(t_{k}^{-}\right), \mathfrak{Z}\left(t_{k}^{+}\right)$and $\mathfrak{Z}\left(t_{k}^{-}\right)$represent the right and left limits of $\mathfrak{Z}(t)$ at $t=t_{k}$ respectively, and they satisfy that $\mathfrak{Z}\left(t_{k}^{-}\right)=\mathfrak{Z}\left(t_{k}\right)$. If $\mathfrak{Z} \in \mathfrak{C}([-\mu, T], \mathbb{R})$, then for any $t \in[0, T]$, define $\mathfrak{Z}_{t}$ by $\mathfrak{Z}_{t}(\theta)=\mathfrak{Z}(t+\theta)$ for $\theta=[-\mu, 0]$, here $\mathfrak{Z}_{t}$ represents the history of the state from time $t-\mu$ to present time $t, \psi \in \mathfrak{C}([-\mu, 0], \mathbb{R})$ and $\psi(0)=0$.
The rest of this paper is organized as follows. In Section 2, we give some notations and recall some concepts and preliminary results. In Section 3, the existence and uniqueness of the problem(1) are obtained by successive approximation method. In Section 4, an example is given to demonstrate the effectiveness of the main results.

\section{Preliminaries}

In this section, we recollect several definitions of fractional derivatives and integals from the papers ${ }^{27-30}$

Definition 4.1 The fractional (arbitrary) order integral of the function $\mathfrak{H} \in L_{1}\left([a, b], \mathbb{R}^{+}\right)$of order $\omega \in \mathbb{R}^{+}$is defined by

$$
I_{a}^{\omega} \mathfrak{H}(t)=\int_{a}^{t} \frac{(t-s)^{\omega-1}}{\Gamma(\omega)} \mathfrak{H}(s) d s,
$$

where $\Gamma$ is the gamma function.

Definition 4.2 For a function $\mathfrak{H}$ given on the interval $[a, b]$, the Caputo fractional order derivative of $\mathfrak{H}$, is defined by

$$
\begin{aligned}
& \left(\begin{array}{l}
c \\
D^{\omega}+{ }^{+} \\
\text {Ho }
\end{array}\right)(t)=\frac{1}{\Gamma(n-\omega)} \int_{a}^{t}(t-s)^{n-\omega-1} \mathfrak{H}^{(n)}(s) d s, \\
& \text { where } n=[\omega]+1 .
\end{aligned}
$$

Definition 4.3 The generalized left-sided fractional integral ${ }^{\rho} I^{\omega}+{ }^{\omega}$ of order $\omega \in \mathfrak{C}(\operatorname{Re}(\omega))$ is defined by

$$
\left({ }^{\rho} I^{\omega} \mathfrak{H}\right)(t)=\frac{\rho^{1-\omega}}{\Gamma(\omega)} \int_{a}^{t}\left(t^{\rho}-s^{\rho}\right)^{\omega-1} s^{\rho-1} \mathfrak{H}(s) d s,
$$$$
\text { for } t>a \text {, if the integral exists. }
$$

Definition 4.4 The generalized fractional derivative, corresponding to the generalized fractional integral (4), is defined for $0 \leq a<t$, by 
$\left({ }^{\rho} D^{\omega} \mathfrak{H}\right)(t)=\frac{\rho^{\omega-n+1}}{\Gamma(n-1)}\left(t^{1-\rho} \frac{d}{d t}\right)^{n} \int_{a}^{t}\left(t^{\rho}-s^{\rho}\right)^{n-\omega-1} s^{\rho-1} \mathfrak{H}(s) d s$,

if the integral exists.

Definition 4.5 The Caputo-type generalized fractional derivative, ${ }^{\rho} D^{\omega}+\mathfrak{H}(t)$ is defined via the above generalized fractional derivative (5) ${ }^{a^{+}}$as follows

$$
\begin{aligned}
& \left({ }_{c}^{\rho} D_{a^{+}}^{\omega} \mathfrak{H}\right)(t)=\left(\begin{array}{r}
\rho \\
D^{\omega} \\
a^{+}
\end{array}\left[\mathfrak{H}(s)-\sum_{k=0}^{n-1} \frac{\mathfrak{H}^{k}(a)}{k !}(\mu-a)^{k}\right]\right)(t),(6) \\
& \text { where } n=\lceil\operatorname{Re}(\omega)\rceil \text {. }
\end{aligned}
$$

Definition 4.6 The generalized fractional derivative in Caputo sense, corresponding to the generalized fractional integral in Caputo sense (6), is defined for $0 \leq a<t$, by

$$
\begin{aligned}
& \left({ }_{c}^{\rho} D_{a^{+}}^{\omega} \mathfrak{H}\right)(t)=\left(t^{1-\rho} \frac{d}{d t}\right)^{n}\left(_{c}^{\rho} I_{a^{+}}^{\omega} \mathfrak{H}\right)(t) \\
& =\frac{\rho^{\omega-n+1}}{\Gamma(n-\omega)}\left(t^{1-\rho} \frac{d}{d t}\right)^{n} \int_{a}^{t}\left(t^{\rho}-s^{\rho}\right)^{n-\omega-1} s^{\rho-1} \mathfrak{H}(s) d s .
\end{aligned}
$$

Remark 4.7 In Caputo sense, the Katugampola fractional derivative operator ${ }_{c}^{\rho} D_{t}^{\omega}$ is a left inverse of the integral operator ${ }_{c}^{\rho} I_{t}^{\omega}$ but in general is not a right inverse,

$$
{ }_{c}^{\rho} D_{t}^{\omega}\left(I_{t}^{\omega} \mathfrak{Z}(t)\right)=\mathfrak{Z}(t)
$$

and the following holds

$$
\rho_{c} I_{t}^{\omega}\left(I_{t}^{\omega} \mathfrak{Z}(t)\right)=\mathfrak{Z}(t)-\sum_{k=0}^{n-1} \frac{\left(t^{\rho}-a\right)^{k}}{k !} \mathfrak{Z}^{(k)}(a), t \in[a, b]
$$

For readers' understanding, we introduce the following notations for the following lemma and theorem.

Let $\mathfrak{J}=[0, T], \quad \mathfrak{J}_{\mathfrak{o}}=\left[0, t_{1}\right], \quad \mathfrak{J}_{i}=\left[t_{i}, t_{i+1}\right], \quad i=1,2,3, \ldots ., m-1$, $\mathfrak{J}_{m}=\left[t_{m}, T\right]$ and $\mathfrak{J}^{\prime}=\mathfrak{J} \backslash\left\{t_{1}, t_{2}, \ldots t_{m}\right\}$.

We denote $\mathfrak{P C}(\mathfrak{J})=\left\{\mathfrak{Z}:[0, T] \rightarrow \mathbb{R} \backslash \mathfrak{Z} \in \mathfrak{C}^{\mathfrak{r}}\left(\mathfrak{J}^{\prime}, \mathbb{R}\right)\right\}, \mathfrak{Z}\left(t_{k}^{+}\right)$and $\mathfrak{Z}\left(t_{k}^{-}\right)$ exist and $\mathfrak{Z}\left(t_{k}^{-}\right)=\mathfrak{Z}\left(t_{k}\right), k=\{1,2, \ldots, m\}$. Obviously, $\mathfrak{P C}(\mathfrak{J})$ is a Banach space with the norm $\|\mathfrak{Z}\|==_{t \in \mathfrak{J}}^{\sup }|\mathfrak{Z}(t)|$.

Lemma 4.8 Assume that $\mathfrak{H} \in \mathfrak{C}(\mathfrak{J}, \mathbb{R}), \quad T>0$. A function $\mathfrak{Z} \in \mathfrak{P C}(\mathfrak{J})$ is a solution of the initial value problem

$$
\left\{\begin{array}{c}
{ }_{c}^{\rho} D^{\omega} \mathfrak{Z}(t)=\mathfrak{H}(t), t \neq t_{k}, t \in \mathfrak{J}:=[0, T] \\
0^{+} \\
\Delta \mathfrak{Z}\left(t_{k}\right)=I_{k}\left(\mathfrak{Z}\left(t_{k}\right)\right), k=1,2, \ldots, m ; \\
\mathfrak{Z}(t)=\psi(t), t \in[-\mu, 0]
\end{array}\right.
$$

if and only if $\mathfrak{Z}$ satisfies the following integral equation

$$
\begin{aligned}
& \text { if and only if } \mathfrak{Z} \text { satisfies the following integral equation } \\
& \qquad \begin{array}{c}
\psi(t), \quad t \in[-\mu, 0] ; \\
\frac{\rho^{1-\omega}}{\Gamma(\omega)} \int_{t_{k}}^{t}\left(t^{\rho}-s^{\rho}\right)^{\omega-1} s^{\rho-1} \mathfrak{H}(s) d s+\sum_{j=1}^{k} I_{j}\left(\mathfrak{Z}\left(t_{j}\right)\right) \\
+\sum_{i=0}^{k-1} \frac{\rho^{1-\omega}}{\Gamma(\omega)} \int_{t_{i}}^{t_{i+1}}\left(t_{i+1}^{\rho}-s^{\rho}\right)^{\omega-1} s^{\rho-1} \mathfrak{H}(s) d s, \quad t \in\left(t_{k}, t_{k+1}\right], k=0,1,2, \ldots, m .
\end{array}
\end{aligned}
$$

Proof. Assume that $\mathfrak{Z}$ satisfies (9). One can see, from Remark 2.7 and $\psi(0)=0$, that

$$
\mathfrak{Z}(t)=\frac{\rho^{1-\omega}}{\Gamma(\omega)} \int_{0}^{t}\left(t^{\rho}-s^{\rho}\right)^{\omega-1} s^{\rho-1} \mathfrak{H}(s) d s, \text { for } t \in \mathfrak{J}_{0}=\left[t_{0}, t_{1}\right] .
$$

In view of $\mathfrak{Z}\left(t_{1}^{+}\right)-\mathfrak{Z}\left(t_{1}^{-}\right)=I_{1}\left(\mathfrak{Z}\left(t_{1}\right)\right)$, we get that

$$
\mathfrak{Z}\left(t_{1}^{+}\right)=I_{1}\left(\mathfrak{Z}\left(t_{1}\right)\right)+\frac{\rho^{1-\omega}}{\Gamma(\omega)} \int_{0}^{t_{1}}\left(t_{1}^{\rho}-s^{\rho}\right)^{\omega-1} s^{\rho-1} \mathfrak{H}(s) d s .
$$

It follows that, for $t \in\left(t_{1}, t_{2}\right]$,

$$
\begin{gathered}
\mathfrak{Z}(t)=\mathfrak{Z}\left(t_{1}^{+}\right)+\frac{\rho^{1-\omega}}{\Gamma(\omega)} \int_{t_{1}}^{t}\left(t^{\rho}-s^{\rho}\right)^{\omega-1} s^{\rho-1} \mathfrak{H}(s) d s \\
=\frac{\rho^{1-\omega}}{\Gamma(\omega)} \int_{t_{1}}^{t}\left(t^{\rho}-s^{\rho}\right)^{\omega-1} s^{\rho-1} \mathfrak{H}(s) d s+\frac{\rho^{1-\omega}}{\Gamma(\omega)} \int_{0}^{t_{1}}\left(t_{1}^{\rho}-s^{\rho}\right)^{\omega-1} s^{\rho-1} \mathfrak{H}(s) d s+I_{1}\left(\mathfrak{Z}\left(t_{1}\right)\right) .
\end{gathered}
$$

In consequence, we can see, by means of $\mathfrak{Z}\left(t_{2}^{+}\right)=\mathfrak{Z}\left(t_{2}^{-}\right)+I_{2}\left(\mathfrak{Z}\left(t_{2}\right)\right)$,

$\mathfrak{Z}\left(t_{2}^{+}\right)=\sum_{i=0}^{1} \frac{\rho^{1-\omega}}{\Gamma(\omega)} \int_{t_{i}}^{t_{i+1}}\left(t_{i+1}^{\rho}-s^{\rho}\right)^{\omega-1} s^{\rho-1} \mathfrak{Z}(s) d s+\sum_{j=1}^{2} I_{j}\left(\mathfrak{Z}\left(t_{j}\right)\right)$,

which implies that for $t \in\left(t_{2}, t_{3}\right]$,

$$
\begin{aligned}
\mathfrak{Z}(t) & =\frac{\rho^{1-\omega}}{\Gamma(\omega)} \int_{t_{2}}^{t}\left(t^{\rho}-s^{\rho}\right)^{\omega-1} s^{\rho-1} \mathfrak{H}(s) d s \\
& +\sum_{i=0}^{1} \frac{\rho^{1-\omega}}{\Gamma(\omega)} \int_{t_{i}}^{t_{i+1}}\left(t_{i+1}^{\rho}-s^{\rho}\right)^{\omega-1} s^{\rho-1} \mathfrak{H}(s) d s+\sum_{j=1}^{2} I_{j}\left(\mathfrak{Z}\left(t_{j}\right)\right) .
\end{aligned}
$$

Repeating the above process, the solution $\mathfrak{Z}(t)$ for $t \in\left(t_{k}, t_{k+1}\right]$ can be written as

$$
\begin{aligned}
\mathfrak{Z}(t) & =\frac{\rho^{1-\omega}}{\Gamma(\omega)} \int_{t_{k}}^{t}\left(t^{\rho}-s^{\rho}\right)^{\omega-1} s^{\rho-1} \mathfrak{H}(s) d s \\
& +\sum_{i=0}^{k-1} \frac{\rho^{1-\omega}}{\Gamma(\omega)} \int_{t_{i}}^{t_{i+1}}\left(t_{i+1}^{\rho}-s^{\rho}\right)^{\omega-1} s^{\rho-1} \mathfrak{H}(s) d s+\sum_{j=1}^{k} I_{j}\left(\mathfrak{Z}\left(t_{j}\right)\right) .
\end{aligned}
$$

Conversely, if $\mathfrak{Z}$ is a solution of (10), one can obtain by a direct computation, that ${ }_{c}^{\rho} D^{\omega} \mathfrak{Z}(t)=\mathfrak{H}(t), t \neq t_{k}, t \in[0, T]$, and $\Delta \mathfrak{Z}\left(t_{k}\right)=\mathfrak{Z}\left(t_{k}^{+}\right)-\mathfrak{Z}\left(t_{k}^{-}\right)=I_{k}\left(\mathfrak{Z}\left(t_{k}\right)\right)$, where

$$
\begin{aligned}
& \mathfrak{Z}\left(t_{k}^{+}\right)=\sum_{i=0}^{k-1} \frac{\rho^{1-\omega}}{\Gamma(\omega)} \int_{t_{i}}^{t_{i+1}}\left(t_{i+1}^{\rho}-s^{\rho}\right)^{\omega-1} s^{\rho-1} \mathfrak{H}(s) d s+\sum_{j=1}^{k} I_{j}\left(\mathfrak{Z}\left(t_{j}\right)\right) \text {, } \\
& \text { and } \\
& \mathfrak{Z}\left(t_{k}^{-}\right)=\frac{\rho^{1-\omega}}{\Gamma(\omega)} \int_{t_{k-1}}^{t_{k}}\left(t_{k}^{\rho}-s^{\rho}\right)^{\omega-1} s^{\rho-1} \mathfrak{H}(s) d s+\sum_{i=0}^{k-2} \frac{\rho^{1-\omega}}{\Gamma(\omega)} \int_{t_{i}}^{t_{i+1}}\left(t_{i+1}^{\rho}-s^{\rho}\right)^{\omega-1} s^{\rho-1} \mathfrak{H}(s) d s \\
& \qquad+\sum_{j=1}^{k-1} I_{j}\left(\mathfrak{Z}\left(t_{j}\right)\right) \text {. } \\
& \text { This completes the proof. }
\end{aligned}
$$

\section{Existence and uniqueness results}

Initially, set $C_{0}=\{v \mid v \in \mathfrak{C}(\mathfrak{J}, \mathbb{R}), v(0)=0\} . \quad$ For each, $v \in C_{0}$, we denote by $\bar{v}$ the function defined by

$$
\bar{v}(t)=v(t), 0 \leq t \leq T \text { and } \bar{v}(t)=0,-\mu \leq t \leq 0 \text {. }
$$

If $\mathfrak{Z}$ is a solution of (1), then $\mathfrak{Z}($.) can be decomposed as 
$\mathfrak{Z}(t)=\bar{v}(t)+\phi(t)$ for $-\mu \leq t \leq T$, which implies that $\mathfrak{Z}_{t}=\bar{v}_{t}+\phi_{t}$ for $0 \leq t \leq T$, where

$$
\phi(t)=0,0 \leq t \leq T \text {, and } \phi(t)=\psi(t),-\mu \leq t \leq 0 .
$$

Therefore, the problem (1) can be transformed into the following fixed point problem of the operator $N: \mathfrak{C}_{0} \rightarrow \mathbb{R}$,

$$
\begin{aligned}
& N v(t)=\frac{\rho^{1-\omega}}{\Gamma(\omega)} \int_{t_{k}}^{t}\left(t^{\rho}-s^{\rho}\right)^{\omega-1} s^{\rho-1} \mathfrak{H}\left(s, \bar{v}_{s}+\phi_{s}\right) d s \\
& +\sum_{i=0}^{k-1} \frac{\rho^{1-\omega}}{\Gamma(\omega)} \int_{t_{i}}^{t_{i+1}}\left(t_{i+1}^{\rho}-s^{\rho}\right)^{\omega-1} s^{\rho-1} \mathfrak{H}\left(s, \bar{v}_{s}+\phi_{s}\right) d s \\
& \quad+\sum_{j=1}^{k} I_{j}\left(\bar{v}\left(t_{j}\right)\right), t \in\left(t_{k}, t_{k+1}\right], k=0,1,2, \ldots, m . \text { (13) }
\end{aligned}
$$

Now, let us present our main results.

Theorem 5.1 For the functions $\mathfrak{H} \in \mathfrak{C}(\mathfrak{J} \times \mathbb{R}, \mathbb{R})$ and $I_{k}: \mathbb{R} \rightarrow \mathbb{R}$, assume the following conditions hold

- There exists a continuous function $\alpha:[0, T] \rightarrow \mathbb{R}^{+}$satisfying $\left|\mathfrak{H}\left(t, p_{t}\right)-\mathfrak{H}\left(t, q_{t}\right)\right| \leq \alpha(t) \sup _{s \in[0, t]}|p(s)-q(s)|, p, q \in \mathbb{R}, t \in[0, T] ;$

- There exists a constant $L_{k}>0$ such that $\left|I_{k}(p)-I_{k}(q)\right| \leq L_{k}|p-q|, k=1,2, \ldots, m ; \quad \sum_{i=1}^{m+1} \frac{\alpha_{i} T^{\rho \omega}}{\rho^{\omega} \Gamma(\omega+1)}+\sum_{j=1}^{m} L_{j}<1$, where $\alpha_{k}=\sup _{t \in\left(t_{k}, t_{k+1}\right)} \alpha(t)$;

- There exists a constant $M>0$ such that $\left|\mathfrak{H}\left(t, \phi_{t}\right)\right| \leq M$, where $\phi$ is defined in (12).

\section{Proof}

To complete the proof, we shall use the method of successive approximations. Define a sequence of functions $v_{n}:[0, T] \rightarrow \mathbb{R}$, $n=1,2, \ldots$ as follows:

$v_{0}(t)=0, v_{n}(t)=N v_{n-1}(t)$. (14)

Since $v_{0}(t)=0$, it is easy to see from $(11)$ that $\left(\bar{v}_{0}\right)_{s}=0$ for $s \in[0, T]$. Thus we have,

$$
\begin{aligned}
& \left|v_{1}(t)-v_{0}(t)\right| \leq \frac{\rho^{1-\omega}}{\Gamma(\omega)} \int_{t_{k}}^{t}\left(t^{\rho}-s^{\rho}\right)^{\omega-1} s^{\rho-1}|\mathfrak{H}(s, \phi(s))| d s+\sum_{j=1}^{k} I_{j}(0) \\
& +\sum_{i=0}^{k-1} \frac{\rho^{1-\omega}}{\Gamma(\omega)} \int_{t_{i}}^{t_{i+1}}\left(t_{i+1}^{\rho}-s^{\rho}\right)^{\omega-1} s^{\rho-1}|\mathfrak{H}(s, \phi(s))| d s \\
& \leq \frac{M\left(t^{\rho \omega}-t_{k}^{\rho \omega}\right)}{\rho^{\omega} \Gamma(\omega+1)}+\sum_{i=1}^{k} \frac{M\left(t_{i}^{\rho \omega}-t_{i-1}^{\rho \omega}\right)}{\rho^{\omega} \Gamma(\omega+1)}+\sum_{j=1}^{k}\left|I_{j}(0)\right| \\
& \leq \sum_{i=1}^{m+1} \frac{M\left(t_{i}^{\rho \omega}-t_{i-1}^{\rho \omega}\right)}{\rho^{\omega} \Gamma(\omega+1)}+\sum_{j=1}^{k}\left|I_{j}(0)\right|:=N_{0}, k=1,2, . ., m, \\
& \text { it follows that }
\end{aligned}
$$$$
\left\|v_{1}(t)-v_{0}(t)\right\| \leq N_{0} .
$$

Furthermore,

$$
\begin{aligned}
& \left|v_{n}(t)-v_{n-1}(t)\right| \leq \frac{\rho^{1-\omega}}{\Gamma(\omega)} \int_{t_{k}}^{t}\left(t^{\rho}-s^{\rho}\right)^{\omega-1} s^{\rho-1}\left|\mathfrak{H}\left(s,\left(\bar{v}_{n-1}\right)_{s}+\phi_{s}\right)-\mathfrak{H}\left(s,\left(\bar{v}_{n-2}\right)_{s}+\phi_{s}\right)\right| d s \\
& +\sum_{i=0}^{k-1} \frac{\rho^{1-\omega}}{\Gamma(\omega)} \int_{t_{i}}^{t_{i+1}}\left(t_{i+1}^{\rho}-s^{\rho}\right)^{\omega-1} s^{\rho-1}\left|\mathfrak{H}\left(s,\left(\bar{v}_{n-1}\right)_{s}+\phi_{s}\right)-\mathfrak{H}\left(s,\left(\bar{v}_{n-2}\right)_{s}+\phi_{s}\right)\right| d s
\end{aligned}
$$

$$
\begin{aligned}
& +\sum_{j=1}^{k}\left|I_{j}\left(\bar{v}_{n-1}\right)\left(t_{j}\right)-I_{j}\left(\bar{v}_{n-2}\right)\left(t_{j}\right)\right| \\
& \leq \frac{\rho^{1-\omega}}{\Gamma(\omega)} \int_{t_{k}}^{t}\left(t^{\rho}-s^{\rho}\right)^{\omega-1} s^{\rho-1} \alpha(s)_{x \in[0, s]}^{\sup }\left|\bar{v}_{n-1}(x)-\bar{v}_{n-2}(x)\right| d s \\
& +\sum_{i=0}^{k-1} \frac{\rho^{1-\omega}}{\Gamma(\omega)} \int_{t_{i}}^{t_{i+1}}\left(t_{i+1}^{\rho}-s^{\rho}\right)^{\omega-1} s^{\rho-1} \alpha(s)_{x \in[0, s]}^{s u p} \bar{v}_{n-1}(x)-\bar{v}_{n-2}(x) \mid d s \\
& +\sum_{j=1}^{k} I_{j}\left|\bar{v}_{n-1}\left(t_{j}\right)-\bar{v}_{n-2}\left(t_{j}\right)\right| \\
& \leq\left(\alpha_{k} \frac{\left(t^{\rho \omega}-t_{k}^{\rho \omega}\right)}{\Gamma(\omega+1)}+\sum_{i=1}^{k} \alpha_{i} \frac{\left(t_{i}^{\rho \omega}-t_{i-1}^{\rho \omega}\right)}{\Gamma(\omega+1)}+\sum_{j=1}^{k} L_{j}\right) \cdot\left\|v_{n-1}-v_{n-2}\right\| \\
& \leq\left(\sum_{i=1}^{m+1} \alpha_{i} \frac{T^{\omega \rho}}{\rho^{\omega} \Gamma(\omega+1)}+\sum_{j=1}^{m} L_{j}\right)\left\|v_{n-1}-v_{n-2}\right\| \\
& :=N_{1}\left\|v_{n-1}-v_{n-2}\right\| \text {, }
\end{aligned}
$$

which implies that $\left\|v_{n}-v_{n-1}\right\| \leq N_{1}\left\|v_{n-1}-v_{n-2}\right\|$ with $N_{1}<1$. Note that for any $r>n>0$, we have

$$
\begin{aligned}
& \left\|v_{r}-v_{n}\right\| \leq\left\|v_{n+1}-v_{n}\right\|+\left\|v_{n+2}-v_{n+1}\right\|+\ldots+\left\|v_{r}-v_{r-1}\right\| \\
& \leq\left(N_{1}^{n}+N_{1}^{n+1}+\ldots+N_{1}^{r-1}\right)\left\|v_{1}-v_{0}\right\| \\
& \left\|v_{r}-v_{n}\right\| \leq \frac{N_{1}^{n}}{1-N_{1}}\left\|v_{1}-v_{0}\right\| . \text { (16) }
\end{aligned}
$$

for sufficiently large numbers $r, n$, it follows from the above inequalities with $N_{1}<1$ that $\left\|v_{r}-v_{n}\right\| \rightarrow 0$. Thus, $\left\{v_{n}(t)\right\}$ is a Cauchy sequence in $\mathfrak{P C}(\mathfrak{J})$. Since $\mathfrak{P C}(\mathfrak{J})$ is a complete Banach space, then $\left\|v_{n}-v\right\| \rightarrow 0 \quad(n \rightarrow \infty)$, for some $v \in \mathfrak{P C}(\mathfrak{J})$, which means that $v_{n}(t)$ is uniformly convergent to $v(t)$ with respect to $t$.

In what follows, we shall show that $v(t)$ is a solution of the equation (1). Observe that

$$
\begin{aligned}
& \left|\frac{\rho^{1-\omega}}{\Gamma(\omega)} \int_{t_{k}}^{t}\left(t^{\rho}-s^{\rho}\right)^{\omega-1} s^{\rho-1} \mathfrak{H}\left(s,\left(\bar{v}_{n}\right)_{s}+\phi_{s}\right) d s-\frac{\rho^{1-\omega}}{\Gamma(\omega)} \int_{t_{k}}^{t}\left(t^{\rho}-s^{\rho}\right)^{\omega-1} s^{\rho-1} \mathfrak{H}\left(s,\left(\bar{v}_{s}+\phi_{s}\right)\right) d s\right| \\
& \quad \leq \frac{\rho^{1-\omega}}{\Gamma(\omega)} \int_{t_{k}}^{t}\left(t^{\rho}-s^{\rho}\right)^{\omega-1} s^{\rho-1}\left|\mathfrak{H}\left(s,\left(\bar{v}_{n}\right)_{s}+\phi_{s}\right)-\mathfrak{H}\left(s,\left(\bar{v}_{s}+\phi_{s}\right)\right)\right| d s \\
& \quad \leq \frac{\rho^{1-\omega}}{\Gamma(\omega)} \int_{t_{k}}^{t} \alpha(t)\left(t^{\rho}-s^{\rho}\right)^{\omega-1} s^{\rho-1} \sup _{x \in[0, s]}\left|\bar{v}_{n}(x)-\bar{v}(x)\right| d s \\
& \quad=\frac{\rho^{1-\omega}}{\Gamma(\omega)} \int_{t_{k}}^{t} \alpha(t)\left(t^{\rho}-s^{\rho}\right)^{\omega-1} s^{\rho-1} \sup _{x \in[0, s]}\left|v_{n}(x)-v(x)\right| d s .
\end{aligned}
$$

Since $v_{n}(t) \rightarrow v(t)$ as $n \rightarrow+\infty$, for any $\varepsilon>0$, there exists a sufficiently large number $n_{0}>0$ such that for all $n>n_{0}$, we have

$$
\left|v_{n}(x)-v(x)\right|<\min \left\{\frac{\rho^{\omega} \Gamma(\omega+1)}{\sum_{i=0}^{m} \alpha_{i} T^{\rho \omega}} \varepsilon, \frac{1}{\sum_{j=1}^{m} L_{j}} \varepsilon\right\} .
$$

Therefore, 


$$
\begin{aligned}
& \left|\frac{\rho^{1-\omega}}{\Gamma(\omega)} \int_{t_{k}}^{t}\left(t^{\rho}-s^{\rho}\right)^{\omega-1} s^{\rho-1} \mathfrak{H}\left(s,\left(\bar{v}_{n}\right)_{s}+\phi_{s}\right) d s-\frac{\rho^{1-\omega}}{\Gamma(\omega)} \int_{t_{k}}^{t}\left(t^{\rho}-s^{\rho}\right)^{\omega-1} s^{\rho-1} \mathfrak{H}\left(s,\left(\bar{v}_{s}+\phi_{s}\right)\right) d s\right|<\varepsilon \\
& \sum_{i=0}^{k-1} \frac{\rho^{1-\omega}}{\Gamma(\omega)} \int_{t_{i}}^{t_{i+1}}\left(t_{i+1}^{\rho}-s^{\rho}\right)^{\omega-1} s^{\rho-1} \mathfrak{H}\left(s,\left(\bar{v}_{n}\right)_{s}+\phi_{s}\right) d s \\
& -\sum_{i=0}^{k-1} \frac{\rho^{1-\omega}}{\Gamma(\omega)} \int_{t_{i}}^{t_{i+1}}\left(t_{i+1}^{\rho}-s^{\rho}\right)^{\omega-1} s^{\rho-1} \mathfrak{H}\left(s,\left(\bar{v}_{s}+\phi_{s}\right)\right) d s \\
& \leq \sum_{i=0}^{k-1} \frac{\rho^{1-\omega}}{\Gamma(\omega)} \int_{t_{i}}^{t_{i+1}}\left(t_{i+1}^{\rho}-s^{\rho}\right)^{\omega-1} s^{\rho-1}\left|\mathfrak{H}\left(s,\left(\bar{v}_{n}\right)_{s}+\phi_{s}\right)-\mathfrak{H}\left(s,\left(\bar{v}_{s}+\phi_{s}\right)\right)\right| d s \\
& \leq \sum_{i=0}^{k-1} \alpha\left(t_{i}\right) \frac{\left(t_{i}^{\rho}-t_{i-1}^{\rho}\right)^{\omega}}{\rho^{\omega}} \Gamma(\omega+1) \sup _{x \in[0, s]}^{\operatorname{su}}\left|v_{n}(x)-v(x)\right| d s<\varepsilon . \\
& \sum_{j=1}^{k} I_{j}\left(\bar{v}_{n}\left(t_{j}\right)\right)-\sum_{j=1}^{k} I_{j}\left(\bar{v}\left(t_{j}\right)\right)\left|\leq \sum_{j=1}^{k} L_{j}\right| \bar{v}_{n}\left(t_{j}\right)-\bar{v}\left(t_{j}\right) \mid \\
& =\sum_{j=1}^{k} L_{j}\left|v_{n}\left(t_{j}\right)-v\left(t_{j}\right)\right|<\varepsilon .
\end{aligned}
$$

In consequence, we can see that for a sufficiently large number $n>n_{0}$,

$$
\begin{aligned}
&|v(t)-N v(t)| \\
& \leq\left|v(t)-v_{n+1}(t)\right|+\left|v_{n+1}(t)-N v_{n}(t)\right|+\left|N v_{n}(t)-N v(t)\right| \\
& \leq\left|v(t)-v_{n+1}(t)\right|+\mid v_{n+1}(t)-\left[\frac{\rho^{1-\omega}}{\Gamma(\omega)} \int_{t_{k}}^{t}\left(t^{\rho}-s^{\rho}\right)^{\omega-1} s^{\rho-1} \mathfrak{H}\left(s,\left(\bar{v}_{n}\right)_{s}+\phi_{s}\right) d s\right. \\
&\left.+\sum_{i=0}^{k-1} \frac{\rho^{1-\omega}}{\Gamma(\omega)} \int_{t_{i}}^{t_{i+1}}\left(t_{i+1}^{\rho}-s^{\rho}\right)^{\omega-1} s^{\rho-1} \mathfrak{H}\left(s,\left(\bar{v}_{n}\right)_{s}+\phi_{s}\right) d s+\sum_{j=1}^{k} I_{j}\left(\bar{v}_{n}\left(t_{j}\right)\right)\right] \mid \\
&+\left|\sum_{i=0}^{k-1} \frac{\rho^{1-\omega}}{\Gamma(\omega)} \int_{t_{k}}^{t}\left(t^{\rho}-s^{\rho}\right)^{\omega-1} s^{\rho-1} \mathfrak{H}\left(s,\left(\bar{v}_{n}\right)_{s}+\phi_{s}\right) d s-\frac{\rho^{1-\omega}}{\Gamma(\omega)} \int_{t_{k}}^{t}\left(t^{\rho}-s^{\rho}\right)^{\omega-1} s^{\rho-1} \mathfrak{H}\left(s, \bar{v}_{s}+\phi_{s}\right) d s\right| \\
& \quad+\mid \sum_{i=0}^{k-1} \frac{\rho^{1-\omega}}{\Gamma(\omega)} \int_{t_{i}}^{t_{i+1}\left(t_{i+1}^{\rho}-s^{\rho}\right)^{\omega-1} s^{\rho-1} \mathfrak{H}\left(s,\left(\bar{v}_{n}\right)_{s}+\phi_{s}\right) d s} \\
& \quad-\sum_{i=0}^{k-1} \frac{\rho^{1-\omega}}{\Gamma(\omega)} \int_{t_{i}}^{t_{i+1}}\left(t_{i+1}^{\rho}-s^{\rho}\right)^{\omega-1} s^{\rho-1} \mathfrak{H}\left(s, \bar{v}_{s}+\phi_{s}\right) d s|+| \sum_{j=1}^{k} I_{j}\left(\bar{v}_{n}\left(t_{j}\right)\right)-\sum_{j=1}^{k} I_{j}\left(\bar{v}\left(t_{j}\right)\right) \mid
\end{aligned}
$$

Thus, in view of the convergence of the two previous and (17)-??, one obtains that $|v(t)-N v(t)| \rightarrow 0$, which implies that $v$ is a solution of (1).

Finally, we prove the uniqueness of the solution. Assume that $v_{1}, v_{2}:[0, T] \rightarrow \mathbb{R}$ are two solutions of (1). Note that

$$
\begin{aligned}
& \left|v_{1}(t)-v_{2}(t)\right| \leq \frac{\rho^{1-\omega}}{\Gamma(\omega)} \int_{t_{k}}^{t}\left(t^{\rho}-s^{\rho}\right)^{\omega-1} s^{\rho-1} \alpha(s)_{x \in[0, s]}^{s u p}\left|\bar{v}_{1}(x)-\bar{v}_{2}(x)\right| d s \\
& \quad+\sum_{i=0}^{k-1} \frac{\rho^{1-\omega}}{\Gamma(\omega)} \int_{t_{i}}^{t_{i+1}}\left(t_{i+1}^{\rho}-s^{\rho}\right)^{\omega-1} s^{\rho-1} \alpha(s)_{x \in[0, s]}^{\sup }\left|\bar{v}_{1}(x)-\bar{v}_{2}(x)\right| d s \\
& \quad+\sum_{j=1}^{k} I_{j}\left|\bar{v}_{1}\left(t_{j}\right)-\bar{v}_{2}\left(t_{j}\right)\right|
\end{aligned}
$$

$$
\leq\left(\sum_{i=1}^{p+1} \frac{\alpha_{i} T^{\omega \rho}}{\rho^{\omega} \Gamma(\omega+1)}+\sum_{j=1}^{p} L_{j}\right)\left\|v_{1}-v_{2}\right\|
$$

According to the conditions $\left(A_{3}\right)$, the uniqueness of the problem (1) follows immediately, which completes the proof.

\section{An illustrative example}

Consider the following Katugampola-type fractional impulsive differential equation with delay of the form

$$
\left\{\begin{array}{c}
\rho_{c} D_{0^{+}}^{\omega} \mathfrak{Z}(t)=\frac{e^{-t}\left|\mathfrak{Z}_{t}\right|}{\left(9+e^{t}\right)\left(1+\mid \mathfrak{Z}_{t}\right) \mid}, t \in[0,1], t \neq \frac{1}{2}, 0<\omega<1 ; \\
\Delta \mathfrak{Z}\left(\frac{1}{2}\right)=\frac{\left|\mathfrak{3}\left(\frac{1^{-}}{2}\right)\right|}{3+\left|\mathfrak{Z}\left(\frac{1^{-}}{2}\right)\right|} \\
\mathfrak{Z}(t)=\psi(t)=\frac{e^{-t}-1}{2},-\mu \leq t \leq 0 .
\end{array}\right.
$$

Let us take, $\omega=\frac{1}{2}, \rho=1, \Gamma(\omega+1)>\frac{4}{10}, \mu$ is a non-negative constant. $\mathfrak{Z}_{t}(\theta)=\mathfrak{Z}(t+\theta)$ for $-\mu \leq \theta \leq 0$ and $0 \leq t \leq 1$.

Set $\mathfrak{H}(t, \mathfrak{Z})=\frac{e^{-t} \mathfrak{Z}}{\left(9+e^{t}\right)(1+\mathfrak{Z})}, I(\mathfrak{Z})=\frac{\mathfrak{Z}}{3+\mathfrak{Z}}$, for $(t, \mathfrak{Z}) \in[0,1] \times[0,+\infty)$.

Now, we can see that

$$
\left|\mathfrak{H}\left(t, p_{t}\right)-\mathfrak{H}\left(t, q_{t}\right)\right|=\frac{e^{-t}}{\left(9+e^{t}\right)} \frac{\left|p_{t}-q_{t}\right|}{\left(1+\left|p_{t}\right|\right)\left(1+\left|q_{t}\right|\right)} \leq \frac{e^{-t}}{\left(9+e^{t}\right)}\left|p_{t}-q_{t}\right|
$$

$\leq \alpha(t)_{s \in[0, t]}^{\sup }|p(s)-q(s)|$,

where $\alpha(t)=\frac{e^{-t}}{\left(9+e^{t}\right)}$ and $\alpha={ }_{t \in[0,1]}^{\text {sup }} \alpha(t)=\frac{1}{10}$, so the condition $\left(A_{1}\right)$ is satisfied.

On the other hand, we get that $|I(p)-I(q)|=\frac{3|p-q|}{(3+p)(3+q)} \leq \frac{1}{3}|p-q|, \quad p, q>0$, which satisfies the condition $\left(A_{1}\right)$ of Theorem 3.1 with $L=\frac{1}{3}$.

By a direct computation, we obtain that

$$
\sum_{i=1}^{m+1} \frac{\alpha_{i} T^{\rho \omega}}{\rho^{\omega} \Gamma(\omega+1)}+\sum_{j=1}^{m} L_{j}=\frac{2}{10} \frac{1}{\Gamma(\omega+1)}+\frac{1}{3}<1
$$

and

$$
\left|\mathfrak{H}\left(t, \mathfrak{Z}_{t}\right)\right|=\frac{e^{-t}}{\left(9+e^{t}\right)} \frac{\left|\mathfrak{Z}_{t}\right|}{\left(1+\left|\mathfrak{Z}_{t}\right|\right)} \leq \frac{e^{-t}}{9+e^{t}} \leq \frac{1}{10}, t \in[0,1] .
$$

As a result, the equations in (20) satisfy all the hypotheses in Theorem 3.1 which guarantees that (20) has a unique solution.

\section{Conclusion}

In this note, the existences of solutions of a Katugampolatype fractional impulsive differential equation with delay were investigated. The successive approximation method was employed to show the existence of solutions. The example reflects the applicability of the proposed method. 


\section{Acknowledgements}

None

\section{Conflict of interest}

Author declares that there is no conflict of interest.

\section{References}

1. Agarwal RP, Benchohra M, Slimani BA. Existence results for differential equations with fractional order and impulses. Mem Differential Equations Math Phys. 2008;44:1-21.

2. Glockle WG, Nonnenmacher TF. A fractional calculus approach to selfsimilar protein dynamics. Biophysical Journal. 1995;68(1):46-53.

3. Hilfer R. Applications of Fractional Calculus in Physics. World Scientific 2000 .

4. Kilbas AA, Srivastava HM, Trujillo JJ. Theory and Applications of Fractional Differential Equations. North-Holland Mathematics Studies. 2006;24:1-523

5. Miller KS, Ross B. An Introduction to the Fractional Calculus and Differential Equations. New York: Wiley; 1993. p. 384.

6. Podlubny I. Fractional Differential Equations. Academic Press, San Diego 1999. p. 340.

7. Sabatier J, Agrawal OP, Machado JAT. Advances in Fractional Calculus Theoretical Developments and Applications in Physics and Engineering. Springer. 2007.

8. Samko SG, Kilbas AA, Marichev OI. Fractional Integrals and Derivatives: theory and applications. USA: Gordon and Breach; 1993.

9. Krasnoselskii MA. Positive Solutions of Operator Equations. The Netherlands: Noordhoff, Groningen; 1964. p. 381.

10. Lakshmikantham V, Bainov DD, Simeonov PS. Theory of Impulsive Differential Equations. Worlds Scientific. 1989;6:288.

11. Samoilenko AM, Perestyuk NA. Impulsive Differential Equations. World Scientific: Singapore; 1995. p. 462

12. Sun JX. Nonlinear Functional Analysis and its Application. Beijing: Science Press; 2008

13. Benchohra M, Henderson J, Ntouyas SK, et al. Existence results for fractional order functional differential equations with infinite delay. J Math Anal Appl. 2008;338(2):1340-1350.

14. Deng J, Qu H. New uniqueness results of solutions for fractional differential equations with infinite delay. Comput Math Appl. 2010;60(8):2253-2259.
15. Lin AH, Ren Y, Xia NM. On neutral impulsive stochastic integrodifferential equations with infinite delays via fractional operators. Math Comput Model. 2010;51(6):413-424.

16. Monch H, Von Harten GF. On the Cauchy problem for ordinary differential equations in Banach spaces, Archiv Math Basel. 1982;39(2):153-160.

17. Benchohra M, Slimani BA. Existence and uniqueness of solutions to impulsive fractional differential equations. Electronic $J$ Differential Equations. 2009;10:1-11.

18. Benchohra M, Djamila Seba. Impulsive fractional differential equations in Banach Spaces. Electronic Journal of Qualitative Theory of Differential Equations. 2009;8:1-14

19. Benchohra M, Soufyane Bouriah. Memoirs on Differential Equations and Mathematical Physics. 2016;69:15-31

20. Henderson J, Ouahab A. Impulsive differential inclusions with fractional order. Comput Math Appl. 2010;59(3):1191-1226.

21. Tian YS, Bai ZB. Existence results for the three-point impulsive boundary value problem involving fractional differential equations. Comput Math Appl. 2010;59(8):2601-2609.

22. Wang GT, Ahmad B, Zhang LH. Some existence results for impulsive nonlinear fractional differential equations with mixed boundary conditions. Comput Math Appl. 2011;62(3):1389-1397.

23. Xiaozhi Zhang, Chuanxi Zhu, Zhaoqi Wu, The Cauchy problem for a class of fractional impulsive differential equations with delay. Electronic Journal of Qualitative Theory of Differential Equations. 2012;37:1-13.

24. Zhang XM, Huang XY, Liu ZH. The existence and uniqueness of mild solutions for impulsive fractional equations with nonlocal conditions and infinite delay. Nonlinear Anal Hybrid Syst. 2010;4(4):775-781.

25. Vivek D, Kanagarajan K, Harikrishnan S. Theory and analysis of impulsive type pantograph equations with Katugampola fractional derivative. Journal of Vibration Testing and System Dynamics. 2018;2(1):9-20.

26. Xiaozhi Zhang, Chuanxi Zhu, Zhaoqi Wu, The Cauchy problem for a class of fractional impulsive differential equations with delay. Electronic Journal of Qualitative Theory of Differential Equations. 2012;37:1-13.

27. Katugampola UN. A new approach to generalized fractional derivatives. Bull Math Anal App. 2014;6(4):1-15.

28. Katugampola UN. New approach to a generalized fractional integral. Appl Math Comput. 2011;218(3):860-865.

29. Katugampola UN. Existence and uniqueness results for a class of generalized fractional differential equations. Bull Math Anal App. 2016.

30. Vivek D, Kanagarajan K, Harikrishnan S. Existence and uniqueness results for pantograph equations with generalized fractional derivative. Jour Nonlinear Anal App. 2017;2:105-112. 